University of Nebraska - Lincoln

DigitalCommons@University of Nebraska - Lincoln

Virology Papers

Virology, Nebraska Center for

10-30-2005

\title{
Suppressors of RNA Silencing Encoded by Plant Viruses and Their Role in Viral Infections
}

\author{
Feng Qu \\ University of Nebraska-Lincoln, qu.28@osu.edu \\ Thomas Jack Morris \\ University of Nebraska-Lincoln, jmorris1@unl.edu
}

Follow this and additional works at: https://digitalcommons.unl.edu/virologypub

Part of the Virology Commons

Qu, Feng and Morris, Thomas Jack, "Suppressors of RNA Silencing Encoded by Plant Viruses and Their Role in Viral Infections" (2005). Virology Papers. 125.

https://digitalcommons.unl.edu/virologypub/125

This Article is brought to you for free and open access by the Virology, Nebraska Center for at DigitalCommons@University of Nebraska - Lincoln. It has been accepted for inclusion in Virology Papers by an authorized administrator of DigitalCommons@University of Nebraska - Lincoln. 
Published in FEBS Letters 579:26 (October 31, 2005), pp. 5958-5964; doi 10.1016/j.febslet.2005.08.041

Copyright (C) 2005 Federation of European Biochemical Societies; published by Elsevier B.V. Used by permission. http://www.sciencedirect.com/science/journal/00145793

Submitted June 20, 2005; revised August 20, 2005; accepted August 21, 2005; published online September 2, 2005. Edited for FEBS Letters by Shou-Wei Ding.

Minireview

\title{
Suppressors of RNA Silencing Encoded by Plant Viruses and Their Role in Viral Infections
}

\author{
Feng Qu and T. Jack Morris \\ School of Biological Sciences, University of Nebraska-Lincoln, \\ E229 Beadle Center, Lincoln, NE 68588-0666, United States \\ Corresponding author-T. J. Morris, fax 402 472-2083, email jmorris@unl.edu
}

\begin{abstract}
RNA silencing as a robust host defense mechanism against plant viruses is generally countered by virus-encoded silencing suppressors. This strategy is now increasingly recognized to be used by animal viruses as well. We present here an overview of the common features shared by some of the better studied plant viral silencing suppressors. We then briefly describe the characteristics of the few reported animal viral suppressors, notably their extraordinary ability of cross-kingdom suppression. We next discuss the basis for biased protection of viral RNA and subviral parasites by silencing suppressors, the link between movement and silencing suppression, the influence of temperature on the outcome of viral infection and the effect of viral silencing suppressors on the microRNA pathway.
\end{abstract}

Keywords: RNA silencing, viral silencing suppressor, virus-host interaction

\section{Introduction}

RNA silencing is a potent surveillance system targeting parasitic RNA in a highly sequence-specific manner, manifesting as post-transcriptional gene silencing in plants or RNA interference in animals. These evolutionarily conserved processes are now known to be operative in most if not all eukaryotic organisms [1]. The RNA silencing pathway in plants presents a formidable defense against viral pathogens. It is becoming increasingly evident that most, if not all plant viruses have adopted counter-defensive strategies to overcome the host silencing pathway. The purpose of this review is to summarize the current understanding of those adaptive strategies with a focus on providing new insight into the molecular basis of viral-host interactions.

Mechanistically, the RNA silencing process in plants can be divided into two distinct stages: initiation and maintenance. At the initiation stage, the host cell senses the presence of doublestranded RNA (dsRNA) as abnormal and uses a dsRNA-specific RNase called Dicer (or DCL for Dicer-like) to digest the dsRNA into a small RNA species of 21-24 nucleotides termed small interfering RNA (siRNA). Although it is often assumed that viral replicative forms provide the dsRNA template recognized by DCLs, it is likely that highly structured regions of the genomic RNA are also primary targets [2,3]. Furthermore, viral RNA may also be converted to a dsRNA target by one of the RNA-dependent RNA polymerases (RdRPs) encoded by the plant host [4]. The siRNA produced by the action of DCLs is then recruited into a RNA-induced silencing complex (RISC), where it mediates the sequence-specific digestion of homologous RNA by RISC. At the maintenance stage, silencing of homologous RNA persists in the absence of the dsRNA trigger. This is accomplished through a siRNA amplification process in which host RdRP synthesizes new dsRNA using siRNA as a primer, and the homologous cellular RNA as template [5]. A unique feature of RNA silencing in plants is that its local induction generates sequence-specific signals that spread systemically throughout the plant $[6,7,9]$. A more detailed description of the RNA silencing machinery can be found in a number of recent reviews $[1,8,10]$.

Our primary focus in this review is on the defensive role of RNA silencing directed against viral infections. It is now well established that plant viruses encode suppressors of RNA silencing to specifically counteract the RNA silencing-based defense mechanism in order to ensure successful systemic invasion of the host plant. Interestingly, virologists have only recently begun to recognize the potentially important role that silencing suppressors may have in modulating virus invasiveness in animal virus infections. The recent review by Voinnet [8] provides a comprehensive list of known virus-encoded silencing suppressors including the few now recognized in animal virus genomes. We have chosen not to duplicate this comprehensive review here, rather we have opted to discuss a limited set of virus-encoded silencing suppressors in an effort to highlight interesting features shared by a few of the better studied suppressors with the intention of hopefully generating some new ideas for future research.

\section{Commonly used approaches for identifying viral suppres- sors of RNA silencing}

The innovative adoption of several experimental approaches has been instrumental in the initial identification of a majority of the now recognized suppressors of RNA silencing encoded by plant viruses, as well as the more recent identification of several animal viral suppressors. An exhaustive review 
of the methodology for suppressor identification has recently been compiled by Moissiard and Voinnet [11]. In the interests of not being duplicative, we have chosen to highlight features of four procedures that have been used most frequently, alone and in combination, to verify the silencing suppression activity of a given viral gene, or to identify multiple suppressors encoded by a single virus [12].

The Agrobacterium infiltration assay (agro-infiltration) has been instrumental in the discovery of a majority of viral silencing suppressors reported so far. This approach has the advantage of permitting relatively facile, inexpensive and highly efficient transient expression of any gene of interest in plant cells $[13,14]$. The suspect virus-encoded gene is first cloned into an appropriate shuttle vector that is transferred to a strain of Agrobacterium tumefaciens. Agrobacterium suspensions designed to deliver the candidate gene and a reporter gene (either green fluorescent protein, GFP or $\beta$-glucuronidase, GUS) into plant cells are usually mixed and pressure-infiltrated into leaves of Nicotiana benthamiana plants. The potential silencing suppressor is identified by the ability of the transiently expressed viral gene to enhance and/or sustain visibly higher levels of expression of the reporter gene. Although this approach has worked very well for the identification of a majority of viral genes with suppressor activity from both plant and animal viral genomes, there are some limitations. Several viral suppressors, including the $2 \mathrm{~b}$ gene of Cucumber mosaic virus (CMV), p25 of Potato virus $X(\mathrm{PVX})$, and the coat protein $(\mathrm{CP})$ of Citrus tristeza virus (CTV), were confirmed by alternative approaches because they displayed very low suppression activity in agro-infiltration assays. It is now thought that these suppressors function primarily to block the signaling of RNA silencing from cells of silencing initiation into surrounding cells and distal parts of the plant. Hence, they are less able to interfere with the RNA silencing pathway in the primary cells in which they are expressed because they would be inclined to trigger silencing targeting their own RNA, thus obscuring their role in silencing suppression.

A second commonly used approach has been to examine whether the expression of a silenced transgene can be restored upon the infection of a particular virus of interest (silencing reversal). The initial assay involves observing if the "reporter" transgene silencing is reversed by the viral infection. Each of the encoded viral genes are then individually tested for their ability to reverse silencing by cloning their cDNAs into an appropriate virus vector which is then used to infect the transgenic plants. PVX has been the most commonly used virus vector owing to its apparent inability to reverse RNA silencing. This method is, however, less reliable and more technically demanding because variables such as plant age and growth conditions can affect the outcome of the experiments. In addition, the approach would fail to detect those viral suppressors that are unable to reverse RNA silencing such as the most obvious example of p25 of PVX [13].

The third procedure we want to mention examines the silencing suppression function of a given viral protein using transgenic plants expressing the test protein. In this assay, the transgenic plants expressing the test protein are genetically crossed with plants carrying a reporter gene that is post-transcriptionally silenced. Progeny are examined for the expression of the reporter gene as a consequence of being released from the silencing. This approach was first used to confirm the silencing suppression ability of the P1/HC-Pro protein of
Tobacco etch potyvirus (TEV) independently by two different groups $[15,16]$. In their experiments, a tobacco transgenic line containing a silenced GUS transgene was found to be resistant to a recombinant TEV strain that also expressed GUS (TEVGUS). The resistance to TEV-GUS, deemed a consequence of RNA silencing, was reversed in progeny resulting from the cross with a tobacco line expressing the P1/HC-Pro, the TEV encoded silencing suppressor. Recently, a similar test system has been developed in Arabidopsis to test several different viral silencing suppressors [17]. Although this approach is considerably more time-consuming than the methods discussed previously, this latest development may prove to be an important tool for research using this model host plant because it has not proved amenable to testing using the agro-infiltration assay.

The fourth procedure we have chosen to mention here involves the use of grafting of plant parts to evaluate the ability of virus-encoded silencing suppressors to hinder the movement of systemic RNA silencing signals. This approach was first used to demonstrate the existence of a systemic silencing signal that moved from the stock portion of a plant silenced for a specific transgene to non-silenced scions expressing the same transgene [6]. Similarly, grafting experiments have been very useful in the identification of the suppressor activity associated with p25 of PVX, and the demonstration that it interferes with the spread of the silencing signal [13]. Graft experiments have also been used to explore the mechanism of silencing suppressor function such as the demonstration that the P1/HC-Pro of TEV does not affect the systemic signaling of silencing [57]. Grafting experiments, however, are generally workable with only larger plants such as tobacco and $N$. benthamiana.

\section{A historical review of some well-studied suppressors}

An interesting feature of the majority of plant viral suppressors characterized to-date is that none share any obvious sequence or structural similarity across viral families and groups. The one common feature shared by many is that they have often been initially identified as pathogenicity determinants or host range determinants. It seems that evolutionary selection of a particular class of viral proteins to function as silencing suppressor bears little relationship to any other primary function the viral protein might have in the virus life cycle. Suppressor activity has been identified in structural (CPs) as well as non-structural proteins involved in almost every viral function including movement proteins, viral replicases, replication enhancers, and transcriptional activators [8]. Hence, investigators are faced with a plethora of potential mechanisms to unravel.

\subsection{P1/HC-Pro encoded by potyviruses}

Studies using TEV as the model system established P1/HCPro as one of the first viral silencing suppressors to be characterized. It was already known at the time that this protein was multifunctional in that it affected aphid transmission, polyprotein processing, genome amplification and the long distance movement of the virus [18]. It was also shown to act as a broad range pathogenicity enhancer causing increased viral RNA accumulation and dramatically more severe viral symptoms in a number of unrelated virus infections [19]. We now know that it strongly suppresses RNA silencing, most likely acting on a 
maintenance step affecting the assembly and/or targeting of the RISC complex $[20,57]$. More recent studies have demonstrated that the role of P1/HC-Pro in genome amplification and long distance movement highly correlates with its silencing suppressor activity. Mutant viruses that lack suppressor activity, while capable of initiating the replication process, are unable to sustain robust amplification and hence display debilitated long distance movement. This shows that suppression of the RNA silencing-based host defense is critical for vigorous viral RNA replication and efficient viral spread throughout infected plants [21]. A breakthrough discovery regarding possible mechanism of silencing suppression was the demonstration of interaction between P1/HC-Pro of TEV and rgs-CaM, a tobacco calmodulin-like protein [22]. These authors showed that rgs-CaM suppresses RNA silencing itself upon over-expression in the plants suggesting that RNA silencing is tightly regulated in plants.

\subsection{The $2 b$ protein of cucumoviruses}

The $2 \mathrm{~b}$ protein of cucumoviruses was recognized as a silencing suppressor at about the same time as P1/HC-Pro of potyviruses [25]. It was initially recognized as a small protein of about 100 amino acids encoded by a cryptic ORF in the viral genome [23]. It was found to enhance the long distance movement of CMV in a host-dependent manner. CMV 2b mutants were capable of systemic invasion of tobacco but not cucumber plants [24]. It was later shown that systemic infection of plants containing a silenced GFP transgene by either CMV or a PVX vector expressing $2 b$ restored GFP expression in the leaves emerging after infection, but not in leaves where GFP silencing had already been established [25]. This and additional studies suggest that CMV $2 \mathrm{~b}$ functions to prevent the systemic spread of RNA silencing signals [26].

\subsection{The $p 19$ of tombusviruses}

Initial experiments with several different tombusviruses, including CNV, Cymbidium ringspot virus, and Tomato bushy stunt virus (TBSV) showed the p19 gene was not essential for cellto-cell movement but functioned to assist systemic spread and symptom development in host plants [27]. Further studies showed that functional p19 was required for systemic invasion in some hosts of TBSV but not others [28], suggesting that this host-dependent requirement of p19 might be important in some as yet undefined antiviral defense of the host plants. It was initially recognized as a suppressor of RNA silencing based on its ability to reactivate expression of a silenced GFP transgene in the systemic leaves of plants infected with either TBSV or PVX carrying a p19 insert [29]. Subsequently, several groups have independently demonstrated the potent silencing suppressor activity of p19 from a number of different tombusviruses using the agro-infiltration assay [30-32].

Impressive progress recently on the structural and functional properties of the p19 makes it now the best characterized of the viral silencing suppressor proteins. Notably, it was the first protein demonstrated to directly bind siRNAs, functioning presumably to prevent the siRNAs from entering the RISC complex [32]. Subsequently, the p19-siRNA complex was crystallized and the structure of the complex resolved [33, 34]. This elegantly established a structural explanation for how dimerization of p19 was essential for binding siRNA. Additional studies by several groups have now verified that the degree of
p19-siRNA binding in vivo correlates with the severity of viral infection [17, 20, 35]. Hence, p19 constitutes the first suppressor for which a target in the silencing pathway has been identified.

\section{4. $p 25$ of $P V X$}

It was initially thought that PVX did not encode a silencing suppressor based on the initial use of the PVX based vector to test for suppressor activity of other viral proteins. However, in a series of elegantly designed experiments, Voinnet et al. [13] later demonstrated that $\mathrm{p} 25$, a protein previously shown to be important in the cell-to-cell movement of PVX, was indeed a silencing suppressor that prevented the movement of silencing signals out of the primary-infected cells in which silencing initiation had taken place. Further, since p25 was able to weakly suppress RNA silencing in the agro-infiltration assay, the authors suggested that the inability of silencing to spread systemically resulted from the ability of p25 to interfere with the production of the mobile silencing signal, a step requiring the involvement of a cellular RNA-dependent RNA polymerase [13]. However, p25 of PVX is a relatively weak suppressor of RNA silencing compared to the p25 equivalents of three other potexviruses, Narcissus mosaic virus, Nandina virus X, and Viola mosaic virus, all of which were able to effectively re-activate a previously silenced GFP transgene [29].

\subsection{Coat protein $(C P)$ of carmoviruses}

Our initial work demonstrating the silencing suppressor activity of TBSV p19 contained an experiment that hinted at a silencing suppression role for the structural protein (CP) of Turnip crinkle virus (TCV) [30]. In that paper, we showed that a TBSV p19 deletion mutant, while capable of systemic movement in N. benthamiana plants, accumulated progressively less viral RNA in the systemic leaves due to loss of silencing suppressor ability. Importantly, when we functionally replaced the TBSV structural protein with the TCV CP to create a chimeric virus, we restored close to wild-type levels of virus accumulation in systemic leaves. This result strongly suggested that the TCV CP not only provided structural protein, but also complemented the silencing suppressor function of TBSV p19. In a subsequent study, we directly demonstrated that TCV CP suppresses RNA silencing strongly using the agro-infiltration assay and also showed that suppression of RNA silencing by TCV CP prevented the accumulation of detectable levels of siRNA in infiltrated leaves. These data suggest that TCV CP functions to interfere with the processing of dsRNA [36]. Our results demonstrating the silencing suppressor activity of TCV $\mathrm{CP}$ was also independently confirmed by Thomas et al. [37] in a sister publication. Since those reports, we have shown that similar suppressor activity is associated with the CP of several other carmoviruses including carnation mottle and cardamine chlorotic fleck viruses.

\subsection{Three different silencing suppressors encoded by Citrus tristeza} virus (CTV)

CTV is a plus sense RNA virus with a genome size of about 20 kilobases $(\mathrm{kb})$ that encodes at least 11 open reading frames. It was recently established that three different proteins, p20, p23 and the CP of this complex virus have silencing suppression activities [12]. Both p20 and p23, but not CP, suppressed 
RNA silencing in the agro-infiltration assay, and were able to reverse transgene silencing. Interestingly, p20 and $\mathrm{CP}$, but not p23 prevented intercellular signaling of RNA silencing. It was suggested that suppression of RNA silencing at multiple steps of the silencing pathway may be essential for viruses with large RNA genomes such as CTV [12].

\subsection{Silencing suppressors encoded by animal viruses}

It is now well established that the RNA silencing machinery is present in animal systems and it likely functions as an important defense against transposons and viruses. It is therefore not unreasonable to expect that animal viruses would have been exposed to similar selection pressure as plant viruses during the course of evolution and would be expected to encode proteins with silencing suppressor activity. It is somewhat surprising, therefore, that definitive reports of animal viral proteins with such suppressor activity are meager compared with reports on plant viruses. Interestingly, the first animal virus suppressor of RNA silencing described is the B2 protein encoded by Flock house virus (FHV), an insect virus known to infect plants $[38,39]$. Subsequently, several dsRNA-binding proteins including the vaccinia virus protein $\mathrm{E} 3 \mathrm{~L}$, and the influenza virus protein NS1 have all been shown to suppress RNA silencing using the plant agro-infiltration system and/or in insect cell cultures [39, 40]. Even more remarkable is the recent demonstration that the Primate foamy virus type 1(PFV-1), a complex retrovirus similar to Human immunodeficiency virus, encodes a potent silencing suppressor (Tas) that functions in both cultured human cells and in Arabidopsis plants [41]. These few examples demonstrate that a systematic search for silencing suppressor activity among the genes of most animal viruses could be very fruitful.

\section{Some common features of virus-encoded silencing suppressors}

\subsection{Cross-kingdom suppression of RNA silencing by viral suppressors}

An interesting feature of most known viral silencing suppressors is that they suppress RNA silencing in both animal and plant cells, regardless of the host origin of the virus. The known suppressors of RNA silencing of animal virus origin, including B2 of FHV, NS1 of influenza virus, E3L of vaccinia virus, and Tas of PFV-1 efficiently suppress RNA silencing in plant systems. Among the few suppressors of plant virus origin that have been tested for silencing suppression activities in animal cell cultures, such as p19 of TBSV, CP of TCV, p15 of Peanut clump virus, only p25 of PVX failed to retain suppressor function $[17,35]$. Given that RNA silencing is a defense mechanism conserved in both animals and plants, it seems logical to suggest that suppressors likely target some conserved components of the respective pathways. It is interesting to note that the characterized protein components of this process share fairly low sequence identity except for conserved amino acid residues within several functionally important regions such dsRNA binding domains and PAZ domains $[42,43]$. Although it cannot be completely ruled out that these conserved domains are targeted by silencing suppressors, it seems more likely that silencing suppressors may more frequently have been selected to target the RNA components of the pathway, such as dsRNA and/or siRNA. Ev- idence supporting this conclusion comes from the known affinity of p19 of TBSV for siRNA [32], the strong RNA binding activity of TCV CP [44], and the established dsRNA binding affinity of NS1 of Influenza virus and E3L of vaccinia virus. Extending on this theme, a recent report regarding the p14 silencing suppressor encoded by Pothos latent virus (PoLV), showed that it bound to both long and short dsRNA, including the siRNA duplex [45]. Interestingly, although the genome of PoLV is similar to other tombusviruses, its suppressor (p14) is a smaller protein than p19 with higher affinity to long dsRNAs. Most recently, the B2 suppressor of FHV has been shown to bind both long and short dsRNA as well, lending additional support for dsRNA and siRNA as main targets of silencing suppressors [58].

\subsection{Biased protection of viral RNA and subviral parasites by silenc- ing suppressors}

Pruss et al. [19] first noted that the P1/HC-Pro protein of TEV preferentially increased the accumulation of the (-)-strand viral RNA of PVX. This suggests that (-)-strand viral RNAs are more susceptible to the RNA silencing-based host defense, whereas (+)-strand viral RNAs are better protected. It would be worthwhile to find out if this observation is more universal for other viruses, and the molecular basis for this interesting differential effect. They also observed that the same suppressor caused an increase in the accumulation level of CMV genomic RNA but not the associated satellite RNA, suggesting that satellite RNAs are more resistant to RNA silencing. Similar results were reported for the defective interfering RNA of TBSV, prompting the conclusion that they too were more resistant to RNA silencing than the helper virus genome [2]. It was suggested that the highly structured nature of these subviral RNAs limited access by the RISC complex and this difference of accessibility accounted for preferential accumulation of the more highly structured smaller RNAs. In this context, the identification of suppressor activity associated with TCV CP provides a possible explanation for the observed role of the $\mathrm{CP}$ in modulating the effects of sat-RNA C on symptom development and satellite RNA accumulation [46].

\subsection{Movement and silencing suppression}

A number of very different suppressor-defective plant virus mutants displayed the similar phenotype of compromised long distance movement that was often host specific. In some extreme cases, such as for PVX p25 and TCV CP [47], the host-specific viral movement defect was even evident at the cell-to-cell level. Now that the silencing suppression activity of these genes has been recognized, it is important to resolve whether the host-dependent movement phenotype is a manifestation of silencing suppression, or some other function associated with these proteins. At least in the case of TEV, a strong correlation between the viral long distance movement and the silencing suppression activity of P1/HC-Pro has been established [21]. There seems to be a similar correlation for TBSV p19 as well [48]. Hence, it would be interesting to learn whether the cell-to-cell movement function associated with both TCV CP and PVX p25 is a manifestation of silencing suppression. This can be examined by attempting to complement their cell-to-cell movement defect with other silencing suppressors. 


\subsection{The abundance of virus-specific siRNAs in infected plants: is it indicative of the strength of silencing?}

Given that siRNAs are the key mediator of RNA silencing, the abundance of siRNAs has been used as an indicator of RNA silencing activity. Some caution is advised in interpreting such data because the level of siRNA present in the host may not always be a reliable indicator of the strength of RNA silencing. First, suppression of RNA silencing by viral suppressors does not always lead to a complete reduction in the accumulation of siRNA. This is evident in the suppression of RNA silencing by two strong and well characterized suppressors, TEV P1/HC-Pro and TBSV p19, both of which do not completely eliminate the accumulation of siRNAs [17, 36]. Secondly, viruses with suppressors that completely eliminate the accumulation of siRNAs in the virus-independent systems, such as TCV CP and PCV p15, may also accumulate high levels of siRNA during the infection of host plants. In fact, it is often observed that virus-specific siRNAs are more abundant in the presence, rather than in the absence, of functional silencing suppressors $[2,13,30]$. Since suppressorless mutants accumulate less viral RNA, the above observation indicates that the level of siRNA correlates with the level of viral RNA in the virus-infected cells. Hence, siRNAs are probably turned over very quickly and the siRNA level most likely reflects ongoing production from viral RNA instead of accumulation. We feel it important to point out this phenomenon because it has implications in interpreting the results of viral infection of plants that are defective in one or more components of the RNA silencing pathways [49]. For example, plants with a defective DCL gene could become more susceptible to viral infection and accumulate more viral RNA due to reduced initial production of siRNAs. However, the accumulation of more viral RNA provides more template for DCLs, and due to the presence of multiple DCL genes with partially redundant functions in the plants, the mutant plants may actually have more siRNAs than virus-infected wildtype plants.

\subsection{The influence of temperature on the outcome of viral infection}

It has long been known that symptoms of most plant viral infections become milder at higher plant growth temperatures. A general explanation for this phenomenon has been offered by Szittya et al. [50], based on the observation that the RNA silencing pathway in plants is less robust at cooler growth temperatures $\left(15^{\circ} \mathrm{C}\right)$ and enhanced at higher temperatures $\left(27^{\circ} \mathrm{C}\right)$. Since the replication of viruses is not generally known to be disproportionately inhibited by higher temperature, one can assume that the activity of viral silencing suppressors is relatively constant over the temperature range that permits viral systemic infection. Thus the level of silencing suppression activity should be relatively steady over the temperature range and therefore more readily overcome at higher temperature due to enhancement of the RNA silencing pathway. Conversely, it can be predicted that at low temperatures, the weakened RNA silencing would be more readily overcome by the viral silencing suppressors. This scenario is supported by our unpublished results showing that some of the genes implicated in the RNA silencing pathway affected viral infection in a temperature-dependent manner. More work is needed to test this and to fully understand the mechanism of temperature-mediated stimulation of RNA silencing.

\subsection{Viral silencing suppressors and the microRNA pathway}

A second RNA silencing pathway mediated by a family of small RNAs termed microRNAs (miRNAs) has been shown recently to be important in developmental gene regulation in both animal and plant systems (for reviews see Refs. [51-53]). The miRNAs are encoded in the genomes of most eukaryotic organisms in the form of large precursor RNA, which are then processed by a DCL into their mature form. The mature miRNAs are similar to siRNAs in size and mode of action, mediating sequence-specific degradation or translational repression of complementary target mRNAs. In plants, most of the miRNA targets are genes that are critical for various developmental processes. A somewhat unexpected, but very interesting property of viral RNA silencing suppressors was observed when they were expressed constitutively in plants as transgenes [54]. Several studies have now shown that transgenic expression of suppressors can alter the accumulation and/or functioning of miRNAs leading to developmental abnormalities related to the action of miRNAs $[17,20]$. Although these observations can be explained as a consequence of the similarities between the siRNA and miRNA-mediated pathways, a better understanding of suppressor function will be needed to fully appreciate the role of suppressors in virus symptom development. It is known that most of the characterized miRNAs assert their roles in the plant meristem, a site where virus invasion rarely occurs. Consequently, the more extreme developmental abnormalities observed in these transgenic plants do not precisely mimic viral symptoms, suggesting that viral suppressors may be exerting their effect on developmental pathways only incidentally during active virus infections. Nevertheless, the viral silencing suppressors are useful tools to dissect both siRNA and miRNA-mediated pathways and the functions of various miRNAs.

\section{Future directions}

The past several years have seen the identification of RNA silencing suppressors from most plant viruses, including both RNA and DNA viruses, and a few animal viruses. Yet for most of these suppressors, the molecular basis of silencing suppression remains unknown. This will surely be a focus area for future research. Challenges ahead include elucidating the mechanisms of viral-mediated silencing suppression for the many types of suppressors that have been discovered. Clearly, identifying potentially novel players in the RNA silencing-based host defense pathway, and better understanding the regulation of the silencing pathway will provide some answers and perhaps new insights into the plant defense systems. A particularly intriguing aspect, as noted recently by Vionnet [8], is the potential connection between the silencing as a defense mechanism and the plant innate immune defense system mediated by disease resistance proteins ( $\mathrm{R}$ proteins). The author noted that at least two silencing suppressor proteins are known to elicit a hypersensitive resistance response. Interestingly, we have demonstrated that the TCV CP suppressor also functions as the primary elicitor of the resistance response in Arabidopsis mediated by the HRT resistance gene [55]. Although our latest results were unable to convincingly link the two pathways [56], the fact that the CP is a key mediator of the defense response in both pathways certainly suggests that there is yet much to be learned about "cross-talk" between these two plant defense systems. Finally, it will be important to explore 
how environmental factors such as light and temperature conditions affect the RNA silencing and symptoms of viral infections. Improved understanding in these areas should ultimately lead to better management of virus diseases.

\section{Acknowledgments}

Reference limitations precluded our ability to cite all of the many appropriate papers pertinent to this topic and we wish to sincerely apologize to those authors for the omission. Our research cited in this review was supported in part by a US Department of Energy Grant (DE-FG02-04ER15531) and by NIH Grant No. P20 RR16469 from the INBRE Program of the National Center for Research Resources.

\section{References}

1. Y. Tomari and P. D. Zamore, Perspective: machines for RNAi, Genes Dev. 19 (2005), pp. 517-529.

2. G. Szittya, A. Molnar, D. Silhavy, C. Hornyik, and J. Burgyan, Short defective interfering RNAs of tombusviruses are not targeted by trigger post-transcriptional gene silencing against their helper virus, Plant Cell 14 (2002), pp. 359-372.

3. A. Molnar, T. Csorba, L. Lakatos, E. Varallyay, C. Christophe Lacomme, and J. Burgyan, Plant virus-derived small interfering RNAs originate predominantly from highly structured single-stranded viral RNAs, J. Virol. 79 (2005), pp. 7812-7818.

4. D. Yu, B. Fan, S. A. MacFarlane, and Z. Chen, Analysis of the involvement of an inducible Arabidopsis RNA-dependent RNA polymerase in antiviral defense, Mol. Plant-Microbe Interact. 16 (2003), pp. 206-216.

5. F. E. Vaistij, L. Jones, and D. C. Baulcombe, Spreading of RNA targeting and DNA methylation in RNA silencing requires transcription of the target gene and a putative RNA-dependent RNA polymerase, Plant Cell 14 (2002), pp. 857-867.

6. J.-C. Palauqui, T. Elmayan, J.-M. Pollien, and H. Vaucheret, Systemic acquired silencing: transgene-specific post-transcriptional silencing is transmitted by grafting from silenced stocks to non-silenced scions, EMBO J. 16 (1997), pp. 4738-4745.

7. O. Voinnet and D. C. Baulcombe, Systemic signaling in gene silencing, Nature 389 (1997), p. 553.

8. O. Voinnet, Induction and suppression of RNA silencing: Insights from viral infections, Nature Rev. Genet. 6 (2005), pp. 206-220.

9. Voinnet, O. (2005) Non-cell autonomous RNA silencing. FEBS Lett. (this issue).

10. Hammond, S. M. (2005) Dicing and slicing - The core machinery of the RNA interference pathways. FEBS Lett. (this issue).

11. G. Moissiard and O. Voinnet, Viral suppression of RNA silencing in plants, Mol. Plant Path. 5 (2004), pp. 71-82.

12. R. Lu, A. Folomonov, M. Shintaku, W.-X. Li, B. W. Falk, W. O. Dawson, and S.-W. Ding, Three distinct suppressors of RNA silencing encoded by a $20-\mathrm{kb}$ viral RNA genome, Proc. Natl. Acad. Sci. USA 101 (2004), pp. 15742-15747.

13. O. Voinnet, C. Lederer, and D. Baulcombe, A viral movement protein prevents spread of the gene silencing signal in Nicotiana benthamiana, Cell 103 (2000), pp. 157-167.
14. L. K. Johansen and J. C. Carrington, Silencing on the spot. Induction and suppression of RNA silencing in the Agrobacterium-mediated transient expression system, Plant Phsiol. 126 (2001), pp. 930-938.

15. K. D. Kasschau and J. C. Carrington, A counter-defensive strategy of plant viruses: Suppression of posttranscriptional gene silencing, Cell 95 (1998), pp. 461-470.

16. R. Anandalakshmi, G. J. Pruss, X. Ge, R. Marathe, A. C. Mallory, T. H. Smith, and V. B. Vance, A viral suppressor of gene silencing in plants, Proc. Natl. Acad. Sci. USA 95 (1998), pp. 13079-13084.

17. P. Dunoyer, C.-H. Lecellier, E. A. Parizotto, C. Himber, and O. Voinnet, Probing the microRNA and small interfering RNA pathways with virus-encoded suppressors of RNA silencing, Plant Cell 16 (2004), pp. 1235-1250.

18. K. D. Kasschau, S. Cronin, and J. C. Carrington, Genome amplification and long-distance movement functions associated with the central domain of tobacco etch potyvirus helper component-proteinase, Virology 228 (1997), pp. 251-262.

19. G. Pruss, X. Ge, X. M. Shi, J. C. Carrington, and V. B. Vance, Plant viral synergism: The potyviral genome encodes a broad-range pathogenicity enhancer that transactivates replication of heterologous viruses, Plant Cell 9 (1997), pp. 859-868.

20. E. J. Chapman, A. I. Prokhnevsky, K. Gopinath, V. V. Dojia, and J. C. Carrington, Viral RNA silencing suppressors inhibit the microRNA pathway at an intermediate step, Genes Dev. 18 (2004), pp. 1179-1186.

21. K. D. Kasschau and J. C. Carrington, Long-distance movement and replication maintenance functions correlate with silencing suppression activity of potyviral HC-Pro, Virology 285 (2001), pp. 71-81.

22. R. Anandalakshmi, R. Marathe, X. Ge, J. M. Herr Jr. , C. Mau, A. Mallory, G. Pruss, L. Bowman, and V. B. Vance, A calmodulin-related protein that suppresses posttranscriptional gene silencing in plants, Science 290 (2000), pp. 142-144.

23. S.-W. Ding, B. J. Anderson, H. R. Haase, and R. H. Symons, New overlapping gene encoded by the cucumber mosaic virus genome, Virology 198 (1994), pp. 593-601.

24. S.-W. Ding, W.-X. Li, and R. H. Symons, A novel naturally occurring hybrid gene encoded by a plant RNA virus facilitates long distance virus movement, EMBO J. 14 (1995), pp. 5762-5772.

25. G. Brigneti, O. Voinnet, W.-X. Li, L.-H. Ji, S.-W. Ding, and D. C. Baulcombe, Viral pathogenicity determinants are suppressors of transgene silencing in Nicotiana benthamiana, EMBO J. 17 (1998), pp. 6739-6746.

26. H. S. Guo and S. W. Ding, A viral protein inhibits the long range signaling activity of the gene silencing signal, EMBO J. 21 (2002), pp. 398-407.

27. M. Russo, J. Burgyan, and G. P. Martelli, Molecular biology of Tombusviridae, Adv. Virus Res. 44 (1994), pp. 381-428.

28. H. B. Scholthof, K.-B. G. Scholthof, M. Kikkert, and A. O. Jackson, Tomato bushy stunt virus spread is regulated by two nested genes that function in cell-to-cell movement and host-dependent systemic invasion, Virology 213 (1995), pp. 425-438.

29. O. Voinnet, Y. M. Pinto, and D. C. Baulcombe, Suppression of gene silencing: a general strategy used by diverse DNA and RNA viruses of plants, Proc. Natl. Acad. Sci. USA 96 (1999), pp. 14147-14152. 
30. F. Qu and T. J. Morris, Efficient infection of Nicotiana benthamiana by Tomato bushy stunt virus is facilitated by the coat protein and maintained by p19 through suppression of gene silencing, Mol. Plant-Microbe Interact. 15 (2002), pp. 193-202.

31. W. Qiu, J.-W. Park, and H. B. Scholthof, Tombusvirus P19mediated suppression of virus-induced gene silencing is controlled by genetic and dosage features that influence pathogenicity, Mol. Plant-Microbe Interact. 15 (2002), pp. 269-280.

32. D. Silhavy, A. Molnar, A. Lucioli, G. Szittya, C. Hornyik, M. Tavazza, and J. Burgyan, A viral protein suppresses RNA silencing and binds silencing-generated, 21-to 25-nucleotide double-stranded RNAs, EMBO J. 21 (2002), pp. 3070-3080.

33. J. M. Vargason, G. Szittya, J. Burgyán, and T. M. T. Hall, Size selective recognition of siRNA by an RNA silencing suppressor, Cell 115 (2003), pp. 799-811.

34. K. Ye, L. Malinina, and D. J. Patel, Recognition of small interfering RNA by a viral suppressor of RNA silencing, $\mathrm{Na}$ ture 426 (2003), pp. 874-878.

35. L. Lakatos, G. Szittya, D. Silhavy, and J. Burgyán, Molecular mechanism of RNA silencing suppression mediated by p19 protein of tombusviruses, EMBO J. 23 (2004), pp. 876-884.

36. F. Qu, T. Ren, and T. J. Morris, The coat protein of Turnip crinkle virus suppresses posttranscriptional gene silencing at an early initiation step, J. Virol. 77 (2003), pp. 511-522.

37. C. L. Thomas, V. Leh, C. Lederer, and A. J. Maule, Turnip crinkle virus coat protein mediates suppression of RNA silencing in Nicotiana benthamiana, Virology 306 (2003), pp. 33-41.

38. H. Li, W. X. Li, and S. W. Ding, Induction and suppression of RNA silencing by an animal virus, Science 296 (2002), pp. 1319-1321.

39. W. X. Li, H. Li, R. Lu, F. Li, M. Dus, P. Atkinson, E. W. Brydon, K. L. Johnson, A. Garcia-Sastre, L. A. Ball, P. Palese, and S. W. Ding, Interferon antagonist proteins of influenza and vaccinia viruses are suppressors of RNA silencing, Proc. Natl. Acad. Sci. USA 101 (2004), pp. 1350-1355.

40. M. O. Delgadillo, P. Sáenz, B. Salvador, J. A. García, and C. Simón-Mateo, Human influenza virus NS1 protein enhances viral pathogenicity and acts as an RNA silencing suppressor in plants, J. Gen. Virol. 85 (2004), pp. 993-999.

41. C.-H. Lecellier, P. Dunoyer, K. Arar, J. Lehmann-Che, S. Eyquem, C. Himber, A. Saib, and O. Voinnet, A cellular microRNA mediates antiviral defense in human cells, Science 308 (2005), pp. 557-560.

42. M. A. Carmell, Z. Xuan, M. Q. Zhang, and G. J. Hannon, The argonaute family: tentacles that reach into RNAi, developmental control, stem cell maintenance, and tumorigenesis, Genes Dev. 16 (2002), pp. 2733-2742.

43. E. J. Finnegan, R. Margis, and P. M. Waterhouse, Posttranscriptional gene silencing is not compromised in the Arabidopsis CARPEL FACTORY (DICER-LIKE 1) mutant, a homolog of Dicer-1 from Drosophila, Curr. Biol. 13 (2003), pp. 236-240.
44. J. M. Skuzeski and T. J. Morris, Quantitative analysis of the binding of turnip crinkle virus coat protein to RNA fails to demonstrate binding specificity but reveals a highly cooperative assembly interaction, Virology 210 (1995), pp. 82-90.

45. Z. Merai, Z. Kerenyi, A. Molnar, E. Barta, A. Valoczi, G. Bisztray, Z. Havelda, J. Burgyan, and D. Silhavy, Aureusvirus P14 is an efficient RNA silencing suppressor that binds double-stranded RNAs without size specificity, J. Virol. 79 (2005), pp. 7217-7226. attenuation by a normally virulent satellite RNA of turnip crinkle virus is associated with the coat protein open reading frame, Plant Cell 7 (1995), pp. 1625-1634.

47. D. L. Hacker, I. T. D. Petty, N. Wei, and T. J. Morris, Turnip crinkle virus genes required for RNA replication and virus movement, Virology 186 (1992), pp. 1-8.

48. M. Chu, B. Desvoyes, M. Turina, R. Noad, and H. B. Scholthof, Genetic dissection of Tomato bushy stunt virus p19-protein-mediated host-dependent symptom induction and systemic invasion, Virology 266 (2000), pp. 79-87.

49. Z. Xie, L. K. Johansen, A. M. Gustafson, K. D. Kasschau, A. D. Lellis, D. Zilberman, S. E. Jacobsen, and J. C. Carrington, Genetic and functional diversification of small RNA pathways in plants, PloS Biol. 2 (2004), pp. 642-652.

50. G. Szittya, D. Silhavy, A. Molnar, Z. Havelda, A. Lovas, L. Lakatos, Z. Banfalvi, and J. Burgyan, Low temperature inhibits RNA silencing-mediated defense by the control of siRNA generation, EMBO J. 22 (2003), pp. 633-640.

51. L. He and G. J. Hannon, MicroRNAs: small RNAs with a big role in gene regulation, Nature Rev. Genet. 5 (2004), pp. 522-531.

52. Wienhold, E. and Plasterk, R. (2005) miRNA function in animal development. FEBS Lett. (this issue).

53. Chen, X. M. (2005) miRNA biogenesis and function in plants. FEBS Lett. 579 (2005); doi 10. 1016/j. febslet. 2005. 07. 071.

54. K. D. Kasschau, Z. Xie, E. Allen, C. Llave, E. J. Chapman, K. A. Krizan, and J. C. Carrington, P1/HC-Pro, a viral suppressor of RNA silencing interferes with Arabidopsis development and miRNA function, Dev. Cell 4 (2003), pp. 205-217.

55. T. Ren, F. Qu, and T. J. Morris, Turnip crinkle virus coat protein binds to and prevents the nuclear localization of an Arabidopsis NAC transcription factor, Virology 331 (2005), pp. 316-324.

56. C.-W. Choi, F. Qu, T. Ren, X. Ye, and T. J. Morris, The RNA silencing suppressor function of Turnip crinkle virus coat protein cannot be attributed to its interaction with the Arabidopsis protein TIP, J. Gen. Virol. 85 (2004), pp. 3415-3420.

57. A. C. Mallory, L. Ely, T. H. Smith, R. Marathe, R. Anandalakshmi, M. Fagard, H. Vaucheret, G. Pruss, L. Bowman, and V. B. Vance, HC-Pro suppression of transgene silencing eliminates the small RNAs but not transgene methylation or the mobile signal, Plant Cell 13 (2001), pp. 571-583.

58. R. Lu, M. Maduro, F. Li, H. W. Li, G. Broitman-Maduro, W. X. Li, and S. W. Ding, Animal virus replication and RNAimediated antiviral silencing in Caenorhabditis elegans, $\mathrm{Na}$ ture 436 (2005), pp. 1040-1043. 